\title{
Physician Burnout \\ and Compassion Fatigue: \\ Individual and Institutional Response to an Emerging Crisis
}

Terri Babineau, MD, FAAFP ${ }^{1, *}$

Ann Thomas, MD, FAAFP ${ }^{2}$

Velyn Wu, MD, FAAFP ${ }^{2}$

\author{
Address \\ *,1 Lynchburg Family Medicine Residency MSt Student in MBCT, University of \\ 0xford, Lynchburg, VA, 24501, USA \\ Email: Terri.Babineau@Centrahealth.com \\ ${ }^{2}$ Lynchburg Family Medicine Residency, Lynchburg, VA, 24501, USA
}

Published online: 9 February 2019

(C) Springer Nature Switzerland AG 2019

This article is part of the Topical Collection on Physician Wellness and Burnout

Keywords Burnout · Compassion fatigue - Institutional factors $\cdot$ EMR $\cdot$ Mindfulness $\cdot$ Cognitive behavioral therapy

\section{Abstract}

Purpose of the review This paper reviews the recent literature in burnout and compassion fatigue focusing on institutional factors influencing burnout where providers work as well as the individual factors affecting providers. In this review, there is an evaluation of the causes and interventions of burnout and compassion fatigue and areas for intervention. Recent findings The percentage of those suffering from burnout is decreasing for the first time in several years, and there are interventions to help decrease the impact of burnout and compassion fatigue. For an individual provider, mindfulness, cognitive behavioral therapy, and improved communication appear to be productive approaches to help with burnout. Institutional approaches that mitigate the impact of burnout in healthcare include assessing provider needs and returning some autonomy and control over workload to the provider.

Summary Burnout and compassion fatigue remain a significant problem in healthcare and impact patient care as well as the well-being of providers. There are approaches that are working to decrease this impact in both the individual provider as well as the institutions in which providers are working. The research in this area from an individual perspective and a business perspective is only beginning. 


\section{Introduction}

The landscape of healthcare is rapidly changing, and burnout is an ever-present problem for today's physicians. An annual survey of physicians in 2017 noted a 4 -year increase in burnout rates of $25 \%$ [1], and the percentage of burnout has just started to decrease for the first time in 2018 [2]. Burnout causes many physicians to retire early, switch careers, or even leave their work altogether [3]. Often, burnout and compassion fatigue are used interchangeably in the literature and compassion fatigue has been defined as "a unique form of burnout" [3] related to both institutional and individual factors; the institutional factors are often described as those beyond the individual practitioner's control and the individual ones are those that have some relation to the individual physician's practice patterns, patient mix, and individual physician personality factors. Interventions to address these factors can be directed to individual physicians and to the organization(s) where they work [4].

\section{What is burnout/compassion fatigue?}

In the 1970s, Freudenberger used the term burnout to describe a problem with psychological health practitioners [5]. Now, burnout is considered an occupational hazard for all of those who work in healthcare. Maslach identified three components to burnout: exhaustion, depersonalization, and reduced personal accomplishment [6]. The three components of burnout may come from various factors in the work or individual's environment. The stress of work that shows in the form of emotional exhaustion and depersonalization is "selfprotective at first-an emotional buffer of concern" [6], thus showing the development of a decreased interpersonal aspect of functioning. The last factor, reduced personal accomplishment, represents the self-evaluation dimension [6].

In 1990, Farber defined three subtypes of burnout among teachers, and this research was expanded to include psychotherapists [7] and now includes healthcare workers. The three subtypes categorize individuals' responses to the frustration that they are feeling with their work and include (1) those who work harder in the face of increasing frustration, (2) those who give up and only do the minimum work required, and (3) those who give up and feel under-challenged which results in them having no interest in their work [7-9]. MonteroMarin et al. have gone on to identify these groups in the following manner: "frenetic or overload" (those working harder), "worn-out or neglect" (the group that gives up), and "underchallenged or lack of development" (those with no interest in their work) [9]. These classifications are being used to identify what changes in work life might be individualized for those experiencing burnout. Those who feel they are not being rewarded in proportion to their efforts have higher rates of burnout [8]. Some personality traits such as type "A" personalities and those who self-identify as workaholics also show risk for increased burnout [10]. Figure 1 provides an artistic view of what burnout looks like to a physician with a type-A personality who feels underchallenged.

Compassion fatigue is a state resulting from "prolonged, continuous and intense contact with patients, the use of self, and exposure to stress" [3]. Compassion fatigue is also described as a secondary traumatic stress syndrome, and there are those who prefer the term secondary traumatic stress syndrome as the wording compassion fatigue may seem to imply that the physician no longer cares [1]. The difference between burnout and compassion fatigue can also be described as burnout being what occurs external to the provider and compassion fatigue/secondary stress is what happens internally to the provider [1].

\section{Why is burnout important?}

Burnout is a cause of decreased productivity and negatively affects the quality of patient care [10]. As burnout rates rise, the rate of physician turnover increases, which leads to significant economic losses considering that the cost to replace one physician is estimated to be between $\$ 500,000$ and $\$ 1,000,000$. This does not include the cost of decreased productivity and patient satisfaction [1012]. Additionally, those providers with higher rates of burnout have higher rates of personal life difficulties such as substance abuse and divorce [13]. 


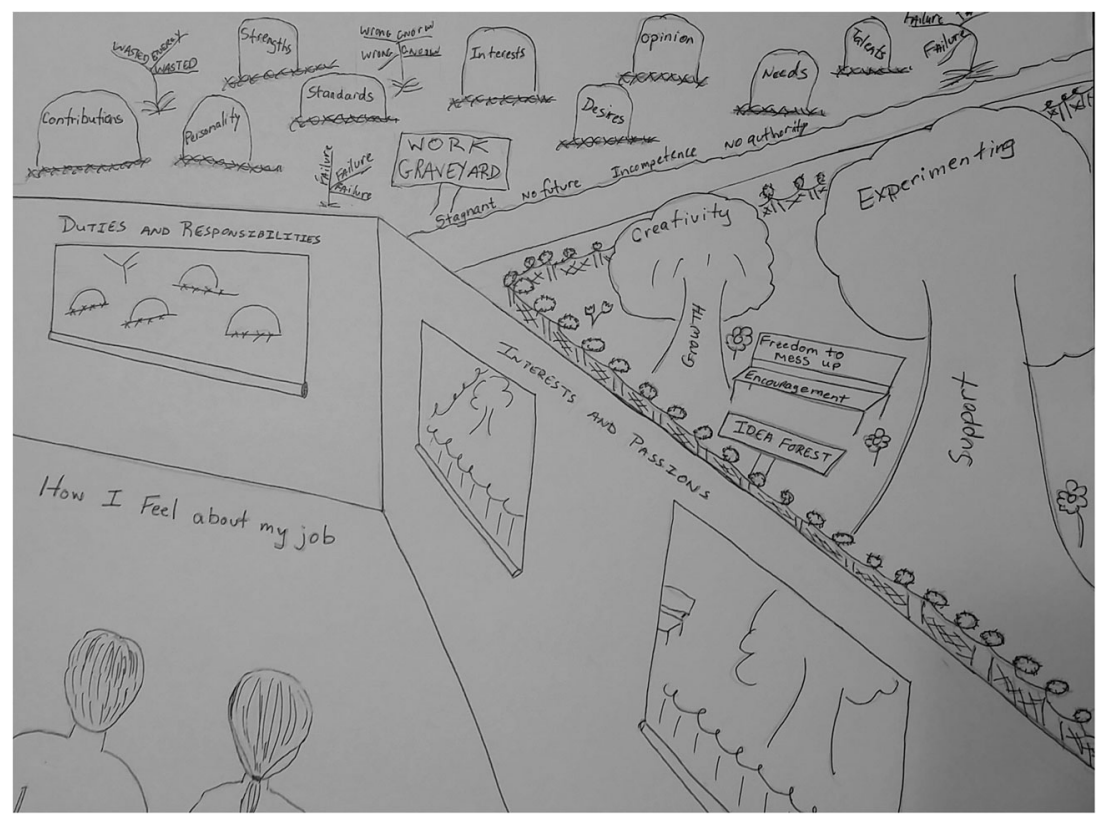

Fig. 1. An artistic view of what burnout looks like to a physician with a type-A personality who feels underchallenged.

Burnout is a large contributor to increased attrition and medical errors [13] and is associated with lower quality of care [12]. Patients with diabetes, hypertension, and other chronic illnesses reported better care and were more likely to adhere to care recommendations when their provider had higher job satisfaction. This shows that "physician wellness and satisfaction are important contributors to patients' adherence to treatment and satisfaction" $[10,11]$. In fact, the importance of provider wellness is so striking to patient safety and care considerations that there are many who have called for a change from the Institute of Healthcare Improvement's Triple Aim of Patient Safety (improving patient care experience, improving population health, and reducing the cost of healthcare per capita) to include physician well-being, creating the Quadruple Aim [14]. It is important to note since the adoption of the Triple Aim, attention in healthcare has shifted from one of public service to a business model with a subsequent larger amount of nonclinical work placed onto physicians resulting in an increase in burnout [13]. Now with this model being accepted in much of the country, the Quadruple Aim is being discussed with the importance of provider wellness added to the list of what is needed for patient safety.
How do we measure burnout and compassion fatigue? The Maslach Burnout Inventory (MBI) is a validated measure of burnout and has become its standard measure $[6,15]$. Burnout is identified with elevated scores in emotional exhaustion and depersonalization and lower scores in personal accomplishment [16]. The measure of the burnout sub-types as identified above (overload, lack of development, and neglect) has been validated against the MBI and is the Burnout Subtype Questionnaire (BCSQ-12) [17]. Another measure used in primary care staff, a Single Item Burnout Measure, has been validated against the subscale of emotional exhaustion of the MBI [18]. The Professional Quality of Life Scale is a 30-item measure used to measure compassion fatigue and potential for burnout. A higher score is associated with a higher risk of compassion fatigue and burnout [19].

\section{Why does burnout occur? (institutional or external factors)}

Some researchers have defined burnout more with dual causation: institutional (external) and individual (internal) causes adding up to the totality of burnout. External factors contributing to burnout include the work environment, career culture, and unending clinical events. For physicians, drivers of physician burnout and engagement have been further categorized into seven 
dimensions: workload and job demands, efficiency and resources, meaning in work, culture and values, control and flexibility, social support and community at work, and work-life integration [20]. Shanafelt and Noseworthy have also specified some of the individual, work unit, organizational, and national factors that influence each dimension [20]. Efficiency and resources, workflows, and processes as well as availability of support staff and allied health professionals influence the physician experience in the workplace [20].

Workload demands include factors such as time pressure, daily workload, conflicting tasks, and productivity expectations. Job control factors include the ability of an individual to make decisions regarding their time management or task completion as well as the ability to influence colleagues $[4,21]$. If the institution where the physician works provides the physician with little control over their work environment or the physician is surrounded by patients who fail to clinically improve, the individual physician may become increasingly fatigued [22]. These situations may also increase the rate of burnout.

Since so many physicians work for large corporations and government entities rather than the small private practices of the past, they can no longer set their own hours, work at their own pace, or have a sense of autonomy in their work [23]. Additionally, physicians work longer hours than most other professions, often working an average of 50-60 h per week for the entirety of their careers with some even working 24 -h shifts $[10,24]$. It has been noted that physicians have a higher rate of burnout than any other profession and primary care physicians are finding that their rates of burnout are among the highest [24] (Fig. 2).

Physicians in past generations had more time for those things in their work lives that provided for their selfgratification like time with patients and control over their work environment [23]. Increasing educational debt, less pay for physicians, increasing productivity requirements requiring physicians to see more patients, and an increasing risk of malpractice contributes to increased stress and burnout for many physicians [23, 25].

Advances in health information technology have also added to the burden of many physicians. As the electronic medical record (EMR) becomes more standard in clinical practice, physicians are feeling increasingly time-burdened. The EMR also allows for easy tracking of quality measures which may not be under the control of the physician [11]. Patient access to the internet where they perceive they find the "correct" diagnosis and use of health portals has created an attitude among many patients that physicians are supposed to perform at almost perfect levels [12]. Most physicians feel that the EMR has not improved the quality of medical care [23]. With these factors that are external to the practicing physician, there is lower job satisfaction as well as increased physician health and personal relationship issues [25].

The culture of medicine also contributes to physician burnout. Early in the medical educational process, physicians learn to deny needing help, not admitting to any

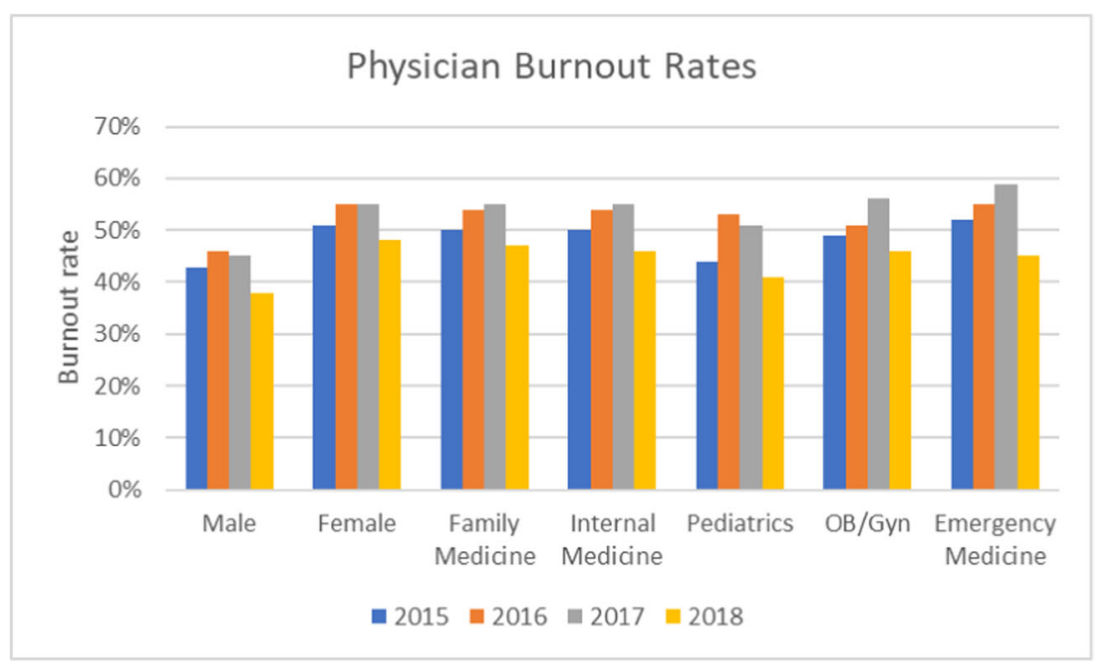

Fig. 2. Data gathered from the Medscape National Physician Burnout and Depression Report 2018; Medscape Lifestyle Report 2017: Race and Ethnicity, Bias, and Burnout; Medscape Lifestyle Report 2016: Bias and Burnout and Medscape Physician Lifestyle Report 2015. 
personal toll that their work takes on them and this idea is re-enforced in their daily work $[26,27]$. Wallace observed that "Doctors often rely on denial and avoidance as coping strategies" and they "are more likely to report a physician with a substance-abuse disorder than one who is emotionally or cognitively impaired" [10]. There is also the perception that there is discrimination by licensing boards against physicians who become ill or burned out which could impair the physician's ability to earn a living if reported [10]. Female physicians may also have more challenges in the area of work-life balance which often contributes to increased family stress [10].

Throughout medical education, physicians are taught to be tough and to always put the needs of the patient first despite great cost to their own health and well-being. Medical students enter their education with the same level of depression and anxiety as others in their age group. Slavin et al. found that by the end of the first year of medical school, 57\% of students had moderate to high symptoms of anxiety and $11.2 \%$ of medical students reported experiencing suicidal ideation [28]. Even with the increasing situation of physician suicide there is little interest in physician mental health among many in medicine [29].

Physicians work in situations that are "cognitively challenging" as they require the highest degree of decision-making and many of these decisions need to be made in an instant. There are few professions where every decision from the tiniest drug adjustment to the most momentous of surgeries could have dire consequences. These kinds of decisions are "life-or-death" and this work is every moment of every day. This kind of cognitive work throughout a workday and workweek can be physically and mentally draining [10].

\section{Why does burnout occur? (individual or internal factors)}

Maslach and Goldberg describe those who suffer from burnout as "feeling drained, cynical, and ineffective" [6]. Using a transactional model showing that there are many factors involved in the process, Fernando \& Consedine included the personal characteristics of an individual physician that may put them at a higher risk [22]. Some physicians are inherently more compassionate, and it may be easier to be compassionate towards some patients (children for instance) and especially to those who show greater appreciation.

Compassion fatigue often shows with individuals having increased anger (especially at work), some deficits in attention span, and increased illnesses [3]. Some physicians who suffer from compassion fatigue report feelings of failure to their profession, sleeplessness, anxiety, and a general lack of confidence [30]. Compassion fatigue may not be from the individual practitioner losing compassion but rather from "unconscious motivations involved in compassion fatigue" [31]. Gerard further discusses that many go into the helping professions to do "over-good" as Freud wrote in Totem and Taboo. "For Freud, these displays of being over-good serve a defensive function, guarding against the anxiety provoked by a prior 'period of badness' that threatened the child's primary relationships" [31]. Looking at compassion fatigue/burnout in this manner may help to explain why so many physicians suffer burnout. If as Freud espoused there is some unconscious driving force to do good and as Fernando and Consedine report that many physicians work in an environment where they have no control over their work environment with patients failing to improve, there is little external reinforcement to feed the physician's internal need to "do good," allowing them to "guard against the anxiety" [22].

The motivational values with which some physicians are raised may also contribute to their level of burnout and compassion fatigue. Those raised in an environment with high expectations but also increased criticism may have higher levels of guilt and shame [32]. A high level of expectations has helped most physicians succeed academically. Perception of failure increases guilt and shame in those with a high level of self-criticism [32]. In healthcare, the stakes are the highest of most professions and failure is not tolerated [10], even if the failure is not actually the failure of the provider but the system or even organizational requirements. There is also a high level of stress for some providers who fear that they will not be able to help patients or even the population where they practice [31]. In these situations, frustration and anxiety increase and the response of the provider may be burnout or compassion fatigue.

\section{What is to be done about burnout: institutional interventions}

The imbalance between job demands and job control may be a trigger for burnout $[4,5,21]$ rather than burnout being a problem of the individual's limitations [12]. Unfortunately, many interventions have focused on increasing the resilience of the individual instead of addressing the workload and control factors [33]. Research studies show that organizational factors such as workload and insufficient personal control contribute to burnout [5, 21]. However, drawing conclusions 
regarding the effectiveness of specific organizationdirected interventions from these studies can be complex. Studies evaluating the effectiveness of organization-directed interventions for physician burnout are comparatively fewer than those characterizing physician burnout, and results of studies of effectiveness of organizational interventions for physician burnout are conflicting $[4,34,35]$ due to the numerous confounding variables surrounding research in this area [4].

Effective organization-directed strategies to address burnout include evaluating workload, autonomy, choice, and fairness [36]. Addressing physician burnout begins with a proper assessment of physician burnout, well-being, and satisfaction. These can be regularly measured as a routine institutional performance metric using standardized instruments [20]. Awareness of factors that influence culture as well as regular assessment to ensure that an organization's actions and values are aligned can strengthen an organization's culture [20]. A recent study of the effects of a primary care transformation initiative on physician burnout and work experience demonstrates a growing understanding that transformational initiatives affect the workplace and physician burnout and suggests a potential benefit to tracking the effects of future organizational initiatives on physician burnout [37].

Harnessing the power of effective physician leadership to promote individual and organizational health by identifying, equipping, and assessing leaders is critical to success. Combining the aforementioned strategies of burnout assessment and leadership, the next strategy then is to develop and implement targeted work unit interventions to improve physician burnout [20]. Other strategies include improved work-life integration, peer support and collaboration, and appropriate use of reward or incentive programs. Work-life balance can be improved by promoting flexibility, reducing work hours [20], and providing physicians with autonomy and choice in an equitable manner $[20,36]$. Providing resources to deliberately foster peer support and greater collaboration between physicians should be offered by each organization [20,38]. Finally, rather than productivity-based physician compensation models (which have been linked to physician burnout due to the vulnerability to overwork), salarybased models or incentive-based models (especially those that include physician well-being) are better options to "provide a safeguard to counter the incentive to overwork" [20,39].

\section{What is to be done about burnout: individual interventions}

In a systematic review of systematic reviews for burnout interventions, it was found that the individual interventions usually included mindfulness techniques, cognitive behavior therapy (CBT), communication improvement, and decreasing stress [4]. These interventions will be reviewed here.

\section{Mindfulness}

It is helpful to understand how mindfulness may work to initiate change in those with burnout/compassion fatigue. "Mindfulness can be described as nonjudgmental attention to experiences of the present moment, including emotions, cognitions, and bodily sensations, as well as external stimuli" [40], and mindfulness can be taught in mindfulness courses or interventions. A cornerstone of mindfulness teaching is compassion for self and others. Mindfulness is based on Buddhist theory (not the religious practice of Buddhism) and a primary outcome of meditation is compassionate response, both for others and almost more importantly in burnout interventions, compassion for one's self [41]. This may be one of the mechanisms that allow for those who study mindfulness to be able to more effectively navigate the current arena of burnout. If Gerard is correct in his assumption that some may suffer from compassion fatigue because of inner conflict over "doing good" and lack and of positive feedback from patients, administration, and the public, then mindfulness may work by negating that effect [31].

There are multiple randomized controlled trials (RCT) that have shown the effectiveness of mindfulness in efforts to mitigate burnout and compassion fatigue. A weekend course of mindfulness intervention for burnout and stress showed improvements in the emotional exhaustion component of burnout, increased mindfulness in the participants, and improvements in stress and depersonalization [42]. Shapiro et al. used mindfulness as an intervention with multiple types of healthcare workers to increase life satisfaction and selfcompassion as well as stress reduction. Using a combination of mindfulness interventions, they showed that there was an increase in self-compassion and the intervention group had greater life satisfaction and decreased burnout [43].

In those with burnout/compassion fatigue, meditation may enable more compassion. Using fMRI to evaluate 
empathy, Laneri et al. showed that when sharing another's suffering the anterior insula (AI), anterior cingulate cortex and the medial prefrontal cortex were all activated. The strength of AI activation is negatively associated with compassion and those in the study with meditation experience had less activation of the left AI [44]. This shows that "current mindfulness meditation could provide an adaptive mechanism in coping with distress due to the empathic sharing of others' suffering, thereby possibly enabling compassionate behavior" [44].

\section{Cognitive behavioral therapy}

In a large review study of interventions for burnout it has been shown that CBT does decrease the symptomatology of burnout and most especially emotional exhaustion in the initial evaluation (usually 1-month follow-up) though this effect was not seen in long-term (up to 1 year) follow-up [45]. In this review of 14 studies, the most common procedure used was CBT with 6 studies using this to combat burnout. The effect did seem to be strongest when CBT was combined with group work though "the individually focused RCT interventions did not produce a statistically significant effect on exhaustion or cynicism" [45]. Thus, CBT may initiate some individual change though the long-lasting effects remain to be seen.

\section{Improved communication}

Improving communication with patients may help physicians to feel more connected with their patients. This interpersonal connection allows physicians to feel that their practice is more humanistic. Following a mandated physician communication 8-h course that emphasized patient communication skills in a large urban-based multidisciplinary practice, "physicians reported significant improvement in measures of empathy and burnout, which were sustained for at least 3 months following the course" [46]. Increasing communication and empathic skills in courses such as this may help physicians to practice with less burnout and more interpersonal connection.

\section{Interventions associated with subtypes}

The intervention for a physician experiencing burnout may need to be individualized as there are too many factors involved in the creation of their burnout [4]. To that end, there has been work done evaluating the three subtypes of burnout as identified previously to begin to determine how an effective intervention could be found. The "frenetic/overload" subtype has a heightened focus on problem-solving and a high level of venting of emotions with the venting having a greater relevance than the problem solving. This group tends to blame others for their own negative feelings and has difficulty with emotional regulation. For this group, emotional regulation may be the starting point to intervene. They may benefit from Acceptance and Commitment Therapy (ACT) helping to increase their "psychological flexibility" [47].

Those with "worn-out/neglect" subtype display behavioral disengagement, have a passive coping style, and are annoyed by monitoring of their activities as it tends to show their shortcomings. They display a lack of dedication and are "immersed in abandonment at work" [47]. This subtype may benefit from some behavioral activation helping to alleviate some of their burnout symptoms before they can even begin to move away from the worn-out symptomatology [47].

The "underchallenged/lack of development" group has cognitive avoidance that displays with "both venting of emotions and behavioral disengagement" [47]. This group is somewhere between overload and neglect, and they show high levels of cynicism. They see the routineness of their work as limiting their development. Because of the increased boredom that this group shows, the authors believe that they may benefit from mindfulness interventions to awaken to everyday experiences [47].

\section{Conclusion}

Burnout and compassion fatigue continue to be major problems in American healthcare and are beginning to be addressed in many institutions across the country. The factors affecting individual providers as well as the factors at the institutions in which they work are associated with the risk of burnout 
development. Institutionally, one of the first actions needs to be acknowledgement of the issue and allocation of resources to adequately address the issues. Organizations should provide resources that encourage individual strategies for physician wellness and burnout prevention. Also, there is a need for changes to the re-imbursement rules, associated changes to electronic charting requirements, and other factors that take providers away from the patient interactions that drew them to medicine. Individually, providers may find improved functioning with mindfulness, cognitive behavioral therapy, and communication initiatives. With continued research into both the types of burnout that providers are experiencing as well as changes to the institutions of American healthcare, burnout may continue to decrease. It is imperative that provider burnout continues to be regarded as a major healthcare consideration.

\section{Compliance with Ethical Standards}

\section{Conflict of Interest}

Terri Babineau declares that she has no conflict of interest. Ann Thomas declares that she has no conflict of interest. Velyn Wu declares that she has no conflict of interest.

Human and Animal Rights and Informed Consent

This article does not contain any studies with human or animal subjects performed by any of the authors.

\section{Publisher's Note}

Springer Nature remains neutral with regard to jurisdictional claims in published maps and institutional affiliations.

\section{References and Recommended Reading}

1. Jablow M. Compassion fatigue: the toll of being a care Provider 2017; Available at: https://news.aamc.org/ medical-education/article/compassion-fatigue-tollbeing-care-provider/. Accessed 10/2/18, 2018.

2. Peckman C. Medscape National Physician Burnout and depression report 2018. Medscape, 2018. https:// www.medscape.com/slideshow/2018-lifestyleburnout-depression-6009235\# 1

3. Ledoux K. Understanding compassion fatigue: understanding compassion. J Adv Nurs. 2015;71(9):204150 .

4. Kalani SD, Azadfallah P, Oreyzi H, Adibi P. Interventions for physician Burnout: a systematic review of systematic reviews. Int J Prev Med. 2018;9(81):1-12.

5. Maslach C, Schaufeli WB, Leiter MP. Job Burnout. Annu Rev Psychol 2001; 2018/05;52(1):397-422.

6. Maslach C, Goldberg J. Prevention of burnout: new perspectives. Appl Prev Psychol. 1998;7(1):63-74.
7. Farber BA. Burnout in psychotherapists: incidence, types, and trends. Psychother Priv Pract. 1990;8(1):3544.

8. Montero-Marín J, García-Campayo J, Mosquera Mera D, López del Hoyo Y. A new definition of burnout syndrome based on Farber's proposal. J Occup Med Toxicol. 2009;4:31.

9. Montero-Marin J, Garcia-Campayo J, Tops M, Manzanera R, Piva Demarzo MM, De Mon MA, GarciaCampayo J. Mindfulness, resilience, and burnout subtypes in primary care physicians: the possible mediating role of positive and negative affect. Front Psychol. 2015;6(DEC).

10. Wallace JE, Lemaire JB, Ghali WA. Physician wellness: a missing quality indicator. LANCET. 2009;374(9702):1714-21.

11. Dyrbye LN, Shanafelt TD, Sinsky CA, Cipriano PF, Bhatt J, Ommaya A, et al. Burnout among healthcare 
professionals a call to explore and address this Underrecognized threat to safe, high-quality care. National Academy of Medicine 2017(July 5, 2017):1-11.

12. Shanafelt T, Goh J, Sinsky C. The business case for investing in physician well-being. JAMA Intern Med. 2017;177(12):1826-32.

13. Anandarajah AP, Quill TE, Privitera MR. Adopting the quadruple aim: the University of Rochester medical center experience. Am J Med. August, 2018;131(8):97986.

14. West CP. Physician well-being: expanding the triple aim. J Gen Intern Med. 2016;31(5):458-9.

15. Maslach C, Jackson S. The measurement of experienced Burnout. J Occup Behav. 1981;2:99-113.

16. Maslach, C, Jackson, SE, Leiter, MP. Consulting Psychologists Press. Maslach burnout inventory. 1986; Available at: https://www.researchgate.net/profile/ Christina_Maslach/publication/277816643_The_ Maslach_Burnout_Inventory_Manual/links/ 5574dbd708aeb6d8c01946d7.pdf. Accessed 18 May 2018.

17. Montero-Marín J, Skapinakis P, Araya R, Gili M, GarcíaCampayo J. Towards a brief definition of burnout syndrome by subtypes: development of the "Burnout clinical subtypes questionnaire" (BCSQ-12). Health Qual Life Outcomes. 2011;9:74.

18. Dolan ED, Mohr DL, Michele J, Sandra F, Stephan D, Nelson KM, et al. Using a single item to measure Burnout in primary care staff: a psychometric evaluation. J Gen Intern Med. 2015;30(5):582-7.

19. Sprang G, Clark JJ, Whitt-Woosley A. Compassion fatigue, compassion satisfaction, and Burnout: factors impacting a Professional's quality of life. J Loss Trauma. 2007;12(3):259-80.

20. Shanafelt TD, Executive Leadership NJH. Physician well-being: nine organizational strategies to promote engagement and reduce Burnout. Mayo Clin Proc. 2017 Jan;92(1):129-46.

21. Awa WL, Plaumann M, Walter U. Burnout prevention: a review of intervention programs. Patient Educ Couns. 2010;78(2):184-90.

22. Fernando AT, Consedine NS. Beyond compassion fatigue: the transactional model of physician compassion. JPS Journal of Pain and Symptom Management. 2014;48(2):289-98.

23. Lister ED, Ledbetter TG, Warren AM. The engaged physician. Mayo Clin Proc. 2015;90:425-7.

24. Shanafelt TD, Boone S, Tan L, Dyrbye LN, Sotile $\mathrm{W}$, Satele D, et al. Burnout and satisfaction with work-life balance among US physicians relative to the general US population. Arch Intern Med. 2012;172(18):1377-85.

25. Dunn PM, Arnetz BB, Christensen JF, Homer L. Meeting the imperative to improve physician well-being: assessment of an innovative program. J Gen Intern Med. 2007;22(11):1544-52.

26. Schwenk TL. Resident depression the tip of a graduate medical education iceberg. JAMA. 2015;314(22):2357.
27. Drummond D. Physician Burnout: its origin, symptoms, and Five Main Causes Fam Pract Manag 2015;22(5).

28. Slavin SJ, Schindler DL, Chibnall JT. Medical student mental health 3.0: improving student wellness through curricular changes. Acad Med. 2014;89(4):573-7.

29. Center C, Davis M, Detre T, Ford DE, Hansbrough W, Hendin $\mathrm{H}$, et al. Confronting depression and suicide in physicians: a consensus statement. JAMA. 2003;289(23):3161.

30. Berg GM, Harshbarger JL, Ahlers-Schmidt CR, Lippoldt D. Exposing compassion fatigue and Burnout syndrome in a trauma team: a qualitative study. J Trauma Nurs. 2016;23(1):3-10.

31. Gerard N. Rethinking compassion fatigue. J Health Org Mgt. 2017;31(3):363-8.

32. Raab K. Mindfulness, self-compassion, and empathy among health care professionals: a review of the literature. J Health Care Chaplain. 2014;20(3):95-108.

33. Gregory ST, Menser T, Gregory BT. An organizational intervention to reduce physician Burnout. J Healthc Manag. 2018;63(5):338-52.

34. Panagioti M, Panagopoulou E, Bower P, Lewith G, Kontopantelis E, Chew-Graham C, et al. Controlled interventions to reduce burnout in physicians: a systematic review and meta-analysis. JAMA Intern Med. 2017;177(2):195-205.

35. Williams D, Tricomi G, Gupta J, Janise A. Efficacy of burnout interventions in the medical education pipeline. Acad Psychiatry. 2015;39:47-54. https://doi.org/ 10.1007/s40596-014-0197-5.

36. Callahan K, Christman G, Maltby L. Battling burnout: strategies for promoting physician wellness. Adv Pediatr. 2018;65(1):1-17.

37. Peikes DN, Swankoski K, Hoag SD, Duda N, Coopersmith J, Taylor EF, et al. The effects of a primary care transformation initiative on primary care physician burnout and workplace experience. J Gen Intern Med. 2018;34:49-57. https://doi.org/10.1007/ s11606-018-4545-0.

38. Wallace JE, Lemaire J. On physician well-being: you'll get by with a little help from your friends. Soc Sci Med. 2007;64(12):2565-77.

39. Scott A, Sivey P, Ait Ouakrim D, et al. The effect of financial incentives on the quality of health care provided by primary care physicians. Cochrane Database Syst Rev. 2011;9:CD008451.

40. Amutio-Kareaga A, García-Campayo J, Delgado L, Hermosilla D, Martínez-Taboada C. Improving communication between physicians and their patients through mindfulness and compassion-based strategies: a narrative review. J Clin Med. 2017;6(3):33.

41. Condon P, Desbordes G, Miller WB, DeSteno D. Meditation increases compassionate responses to suffering. Psychol Sci. 2013;24(10):2125-7.

42. Schroeder DA, Stephens E, Stephens E, Colgan D, Hunsinger M, Christopher MS, et al. Brief mindfulnessbased intervention for primary care physicians: a pilot 
randomized controlled trial. Am J Lifestyle Med. 2018;12(1):83-91.

43. Shapiro SL, Astin JA, Bishop SR, Cordova M. Mindfulness-based stress reduction for health care professionals: results from a randomized trial. Int J Stress Manag. 2005;12(2):164-76.

44. Laneri D, Krach S, Paulus FM, Kanske P, Schuster V, Sommer J, et al. Mindfulness meditation regulates anterior insula activity during empathy for social pain empathy for social pain and mindfulness. Hum Brain Mapp. 2017;38(8):4034-46.

45. Ahola K, Toppinen-Tanner S, Seppanen J. Interventions to alleviate burnout symptoms and to support return to work among employees with burnout: systematic review and meta-analysis. Burnout Research. 2017;4:1-11.

46. Boissy A, Windover AK, Bokar D, Karafa M, Neuendorf K, Frankel RM, et al. Communication skills training for physicians improves patient satisfaction. J Gen Intern Med. 2016;31(7):755-61.

47. Montero-Marin J, Prado-Abril J, Piva-Demarzo MM, Gascon S, García-Campayo J, Gozde O. Coping with stress and types of Burnout: explanatory power of different coping strategies. PLoS One. 2014;9(2):e89090. 